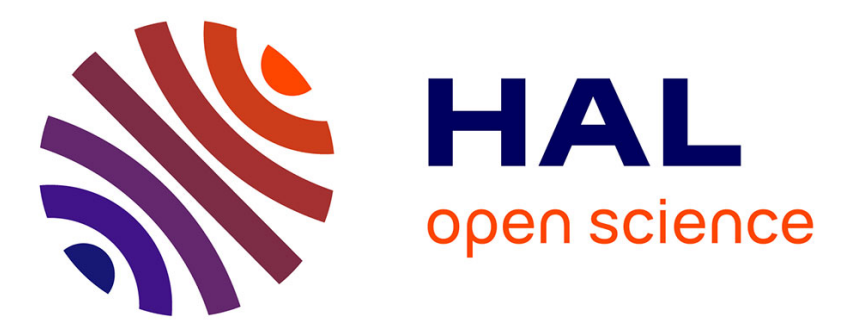

\title{
Data dissemination algorithms for communicating materials using wireless sensor networks
}

Kais Mekki, William Derigent, Ahmed Zouinkhi, Eric Rondeau, Mohamed Naceur Abdelkrim

\section{- To cite this version:}

Kais Mekki, William Derigent, Ahmed Zouinkhi, Eric Rondeau, Mohamed Naceur Abdelkrim. Data dissemination algorithms for communicating materials using wireless sensor networks. The 2nd IEEE International Conference on Future Internet of Things and Cloud, FiCloud'2014, Aug 2014, Barcelona, Spain. hal-01081929

\section{HAL Id: hal-01081929 \\ https://hal.science/hal-01081929}

Submitted on 12 Nov 2014

HAL is a multi-disciplinary open access archive for the deposit and dissemination of scientific research documents, whether they are published or not. The documents may come from teaching and research institutions in France or abroad, or from public or private research centers.
L'archive ouverte pluridisciplinaire HAL, est destinée au dépôt et à la diffusion de documents scientifiques de niveau recherche, publiés ou non, émanant des établissements d'enseignement et de recherche français ou étrangers, des laboratoires publics ou privés. 


\title{
Data Dissemination Algorithms for Communicating Materials using Wireless Sensor Networks
}

\author{
Kais Mekki $^{1,2,3}$, William Derigent ${ }^{1,2}$, Ahmed Zouinkhi $^{3}$, Eric Rondeau ${ }^{1,2}$, and Mohamed Naceur Abdelkrim ${ }^{3}$ \\ ${ }^{1}$ Université de Lorraine, CRAN, UMR 7039, 2, avenue de la forêt de Haye, \\ Vandoeuvre-lès-Nancy Cedex, 54516, France. \\ ${ }^{2}$ CNRS, CRAN, UMR 7039, France. \\ ${ }^{3}$ Research Unit of Modeling, Analysis and Control of Systems (MACS), \\ National Engineering School of Gabes, rue Omar Ibn Elkhattab, 6029 Gabes, Tunisia.
}

\{kais.mekki8,william.derigent,eric.rondeau $\} @$ univ-lorraine.fr \{ah med.zouinkhi,naceur.abdelkrim\}@enig.rnu.tn

\begin{abstract}
The communicating material is a new paradigm of Internet of Things. It is designed to perform efficient product control and ensure an information continuum all along the product life cycle. Therefore, storage of life cycle information and data dissemination in communicating materials are very important issues. This paper provides solutions for storing data on the material by systematic dissemination through integrated ultra-small wireless sensors nodes using counter-based broadcasting scheme, hop-counter and probabilistic storage mechanisms. Different algorithms are developed for nonlocalized and localized dissemination. The performances of our solutions are evaluated for non-segmented and then segmented data. Comparison results between storage mechanisms via simulation using Castalia/OMNeT++ show that probabilistic algorithm provides uniform and efficient data dissemination than hop-counter one for different density level.
\end{abstract}

Keywords-communicating materials; wireless sensor networks; data dissemination

\section{INTRODUCTION}

The communicating material is a new paradigm of industrial information system presented and discussed for the first time in [1]. It enhances a classic material with the following capabilities: it can store data, communicate information at any point of its surface, and keep these previous properties after physical modifications. This concept leads to an important change in the internet of things. Indeed, the material does not communicate using some tags or nodes in specific points, but becomes intrinsically and continuously communicating. To meet this vision, many ultra-small electronic devices (thousands) are inserted into the material of the product during its industrial manufacturing.

The first works focusing on communication materials are presented in $[2,3]$. It introduces a communicating material (etextile) obtained by scattering a huge amount of RFID $\mu$ tags (1500 tags $/ \mathrm{m}^{2}$ ) in a manufactured textile (i.e. $\mu$ tags are tags manufactured by the Hitachi company, measuring $0.15 \mathrm{~mm} \times 0.15 \mathrm{~mm}$ ). The system involves a RFID reader/writer connected to a relational database that contains all the product life cycle information. At each writing operation, the database is explored to select the relevant data items (frag ments of the database tables) that must be stored in the material. To do so, each data item is assigned an importance level between 0 and 1 ( $1=$ highly critical data item, $0=$ ordinary data item), computed via a multi-criteria decision-making algorith $m$. Data ite $m s$ with the highest importance levels are then stored in the $\mu$ tags when the textile passes under the writer module during the manufacturing phases. Since the RFID are memoryconstrained, the data item is splitted (segmented) and stored over several tags using a specific protocol header which is also able to rebuild the initial information. This division process is called segmentation and the resulting parts are segments ${ }^{1}$.

With such system, the data storage in the communicating material requires a reader/writer connection with each tag. If a tag is not connected during the dissemination phase, it will be isolated and left empty which limits the use of RFID technology in solid and large materials such as concrete and wood. Therefore, our current works propose to use wireless sensor networks (WSN) in such products by dispersing ultrasmall and micro (even nano [4]) sensor nodes into the material (e.g. 100 nodes $/ \mathrm{m}^{2}$ ).

This paper presents dissemination algorithms to store relevant data items in wireless sensor nodes scattered into large scale products. Different storage modes are envisaged as shown in figure 1: non-localized storage (i.e. information is spread all over in the whole material) and localized storage (i.e. information is located in a limited region of the material).

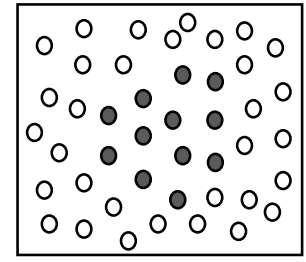

(a) Localized storage

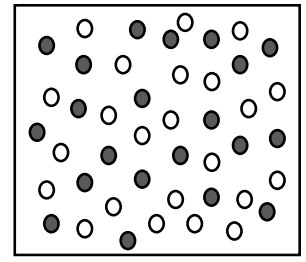

(b) Non-localized storage
Fig. 1. Storage modes for data item dissemination in the material.

\footnotetext{
${ }^{1}$ In our article, the term segmentation is not employed in its classic meaning, usually used for the transport layer of the OSI model.
} 
In the non-localized mode, the algorith m should respect the following constraint: data must be readable everywhere on the material even after a shape transformation (e.g. cutting, sawing, and drilling). So, a given data item should be replicated in a uniform way. Indeed, in figure 2(a), the information is not uniformly disseminated, so pieces of the material are empty and information cannot be read after cutting as example. However, figure 2(b) shows a uniform replication where the information could be read in each piece.

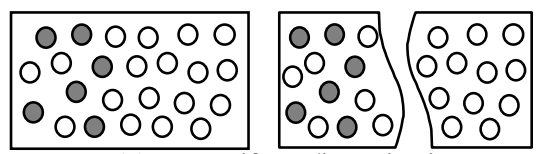

(a) Non-uniform dissemination

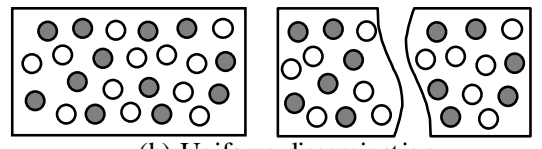

(b) Uniform dissemination

Fig. 2. Uniform dissemination for anticipation of mat erial transformation.

Furthermore, to disseminate information, user sends data item coupled with an importance level. So, the number of nodes storing this data should depend on this level as shown in figures 3(a) and 3(b). Therefore, the dissemination algorithm has to keep the uniformity of data item replication in the material whatever the importance level.

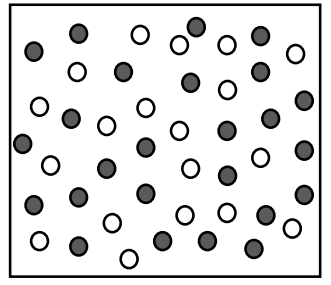

(a) Import ance $=0.6$

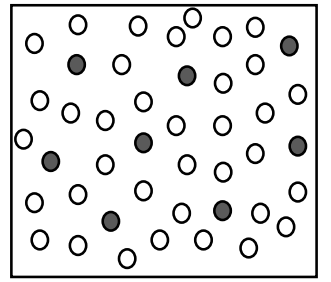

(b) Import ance $=0.1$
Fig. 3. Storage density according to the importance level.

The proposed algorithms are simulated with Castalia/OMNeT++ using a realistic collision model. They are evaluated by studying the uniformity of replication for segmented and non-segmented data items of different importance levels.

The paper is organized as follow. Section 2 is dedicated to related works. Section 3 details the design of our algorithms for data dissemination in the communicating materials. Section 4 is dedicated to the presentation of simulation results and performance evaluation. Finally, section 5 concludes the paper.

\section{RELATED WORK}

In WSN, data dis semination protocols are used for network reprogramming, routing path discovery (e.g. DSR and AODV), fault tolerance, data mules, and mobile sink management [5]. In the past years, various algorithms have been proposed [6]. There are two main approaches: reactive and proactive which is divided into structured and unstructured distribution strategies as shown in figure 4. In the first one, nodes react to an event (e.g. the presence of mules and mobile sink) by disseminating data towards the nodes that are located close to the event positions. In the proactive approach, however, nodes distribute their data towards all or subset of nodes that have the role of storage unit, in anticipation of future event, nodes failure, mule collection, and others. Under structured dissemination, these storage nodes typically form a virtual structure (e.g. grid, line, and rail) within the WSN that make the data available to be retrieved later (e.g. by a mobile sink visiting the nodes to collect data). However in unstructured dissemination, the data is replicated throughout the whole network.

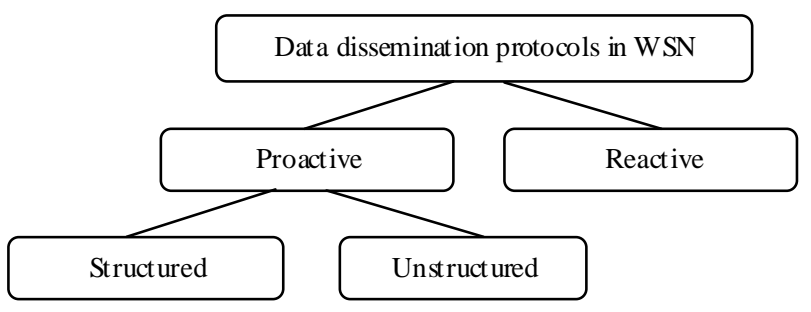

Fig. 4. Classification of data dissemination protocols in WSN.

The aim of this work is to fully disseminate and to uniformly replicate data in the whole communicating material, in order to ease the reading process at any point of the material and to anticipate possible physical transformations. As a result, this section focuses on the protocols related to unstructured proactive dissemination.

Unstructured proactive dissemination protocols in WSN include a broadcast mechanism and a storage strategy, both detailed in the following.

\section{A. Broadcast mechanisms}

Broadcast algorithms are usually referred to as flooding. Flooding is an important algorithm in WSN and is applied when a source node has to send information to subset or all nodes in the network. This is achieved by broadcasting a packet to the entire neighborhood. Each node that receives the packet rebroadcasts it, if it has not been forwarded previously. In this way, the information traverses the whole network and reaches all the nodes which then decide to store it or not according to the storage strategy. Although flooding is a very simple and efficient for data dissemination, it has some deficiencies. The main problems are: duplication (i.e. a node is forced to get information twice from two different nodes), collision (i.e. the broadcast increases the contention), and resource blindness (i.e. nodes do not adopt energy saving mechanisms) [7]. As a consequence, various schemes for controlled flooding have been proposed including probabilistic-based also referred to as gossiping [8] [9], counter-based [10] [11], distance-based and location-based [12].

Distance and location based schemes exploit distance between nodes and their position information. Therefore, nodes need to be equipped with a Received Signal Strength Indicator (RSSI) or a Global Positioning System (GPS). Such additional functions could not be applied in communicating material since the nodes are embedded in the product with a high density (i.e. nodes are very close).

Authors in [13] [14] show that the counter-based scheme outperforms the probabilistic one in terms of reliability (i.e. the percentage of nodes that are reached on average) and efficiency (i.e. the average amount of resources required for broadcasting 
a message). This mechanism can reduce the number of retransmitting nodes with a high arrival rate. Furthermore, it doesn't require specific hardware such as distance and location based schemes. For these reasons, the counter-based strategy is adopted in our dissemination protocol as broadcast mechanism.

\section{B. Storage strategies}

Storage strategies in unstructured proactive dissemination protocols are developed in the literature according to the target application. In [15] [16], the information is replicated in each node for network reprogramming. If a node does not receive the new version of binary code, its software is not updated and is isolated. Authors in [17] [18] [19] propose storage strategies to improve network resilience against the risk of nodes failures. The storage nodes are selected according to some critical parameters such as connectivity, available memory and remaining energy. DEEP [20] adopts another storing strategy for an effective data collection by mobile sink with uncontrolled trajectory. Every node that receives a data stores it with a probability $P$ according to the number of information that must be replicated in each node. In [21], authors use a hopcounter mechanism to replicate data for mobile sink collection. Hop-counter is a process in which a data is repeatedly forwarded from a node to one of its chosen neighbors. At the same time, the number of hops realized from the source node is count. When the counter is equal to a predefined value, the information is stored in the current node.

\section{Synthesis and proposed solution}

The main broadcast and storage approaches for data dissemination protocols in WSN could be summarized in two categories as shown in table 1.

TABLE I. BROADCAST AND STORAGE APPROACHES

\begin{tabular}{|c|c|c|}
\hline & Broadcast mechanism & Storage strategy \\
\hline 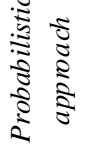 & $\begin{array}{c}\text { Probabilistic-based [8] } \\
\text { [9] }\end{array}$ & $\frac{\text { Probabilistic-storage }[17]}{[18][20]}$ \\
\hline 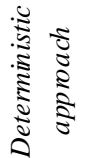 & $\underline{\text { Counter-based [10] [1 }}$ 1] & Hop-counter [19] [21] \\
\hline
\end{tabular}

The above storing strategies use the physical nodes parameters (e.g. memory and energy), network topology, and neighborhood awareness to replicate data throughout the WSN. They don't assume the properties and the characteristic of the disseminated data itself. This problem is the key contribution of our work, in which a dynamic information-based dissemination protocol is proposed and applied on the communicating material framework.

In this current paper, the data importance level is processed. The item with a high level must be replicated more than the lower one. The dissemination algorithm has to ensure the uniformity of replication for each level.
In the literature, few works try to compare data dissemination protocols in the whole wireless sensor network, and, to our knowledge, the replication uniformity is not studied. For these reasons, probabilistic and hop-counter approaches coupled with counter-based flooding are developed in this article and the results are compared from a uniformity point-of-view. The rest of the paper details the proposed data dissemination algorithms, and then presents simulation results obtained for different data item importance level.

\section{DAT A DISSEMINATION ALGORITHMS}

This section presents our different strategies and algorithms of data item dissemination. Firstly, the concept of master node is defined, then the counter-based forwarding scheme is described, and finally the dissemination algorithms are presented.

\section{A. Master Node}

When the user has to disseminate information, he connects to the communicating material through a selected node in its transmission range which is named in this paper "master node". Master node is responsible for forwarding user requests, collecting response messages from all nodes, and disseminating data items. Some constraints could be used to select the mater node such as the highest residual energy level.

\section{B. Broadcast strategy : counter-based scheme}

Our dissemination protocol adopts the counter-based forwarding scheme. When node receives a new packet, it fixes a random waiting delay before making the forwarding decision. During this delay, the sensor counts the number of retransmissions of the same packet by neighbor nodes. After the waiting delay has elapsed, the packet is only forwarded if the number of retransmissions $N$ is smaller than a predetermined threshold. As a result, the forwarding ratio depends on the nodes density: in low density areas this ratio will be high, whereas in high density areas it will be low. Different methods could be used for threshold selection such as those described in [22] [23]. In [22], a node sets the value of the counter threshold according to the number of its neighbor nodes. Similarly, in [23], a node sets it according to the distance from the broadcasting node to itself. Authors in [24] show that a threshold between 4 to 6 retransmissions is preferable from the viewpoint of the trade-off between reliability and efficiency. Moreover, simulation results in [14] [25] show that if the threshold is 4 or more, the reliability is over $99.5 \%$. As the communicating material has high density embedded sensor nodes (e.g. 100 nodes $/ \mathrm{m}^{2}$ ), a threshold of 4 is used for the counter-based scheme of our dissemination algorithms.

\section{Storage strategies}

This section presents two approaches for data item replication in the entire material using the importance level as a parameter. These are respectively hop-counter based and probabilistic-based.

Let us remind that a data item is coupled with an importance level. One part of the storing strategy thus consists in converting this level into a dissemination level computed once by the master node and sent to the sensor network. As a result, for each approach, a conversion function $F$ is defined. 
TABLE II. BROADCAST AND STORAGE APPROACHES

\begin{tabular}{|c|c|c|}
\hline & Hop-counter based & Probabilistic based \\
\hline 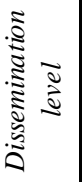 & $\begin{array}{c}H_{0} \\
\text { number of hops bet ween two } \\
\text { storing nodes }\end{array}$ & $\begin{array}{l}P, \\
\text { probability to store the data } \\
\text { item }\end{array}$ \\
\hline 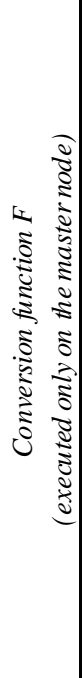 & $\begin{array}{l}\text { Number of } \\
\text { hops } \\
\text { MaxHop } \\
\text { - I: import ance level of the data } \\
\text { item } \\
\text { - MaxHop: the maximal number } \\
\text { of hops needed to reach all the } \\
\text { nodes of the material }\end{array}$ & $\begin{array}{l}\text { With: } \\
\text { - I: import ance level of the data } \\
\text { item }\end{array}$ \\
\hline 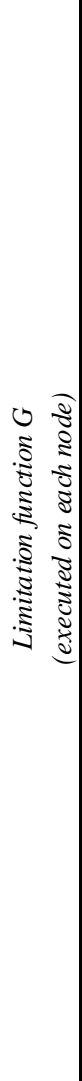 & 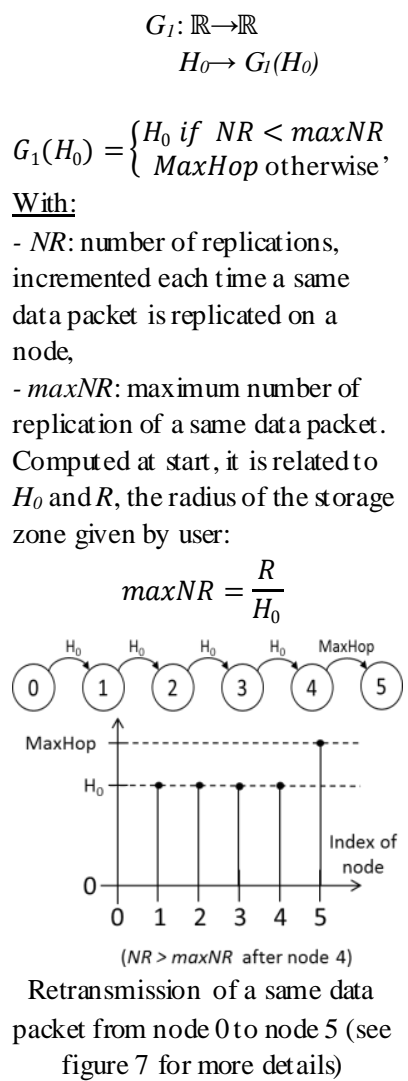 & 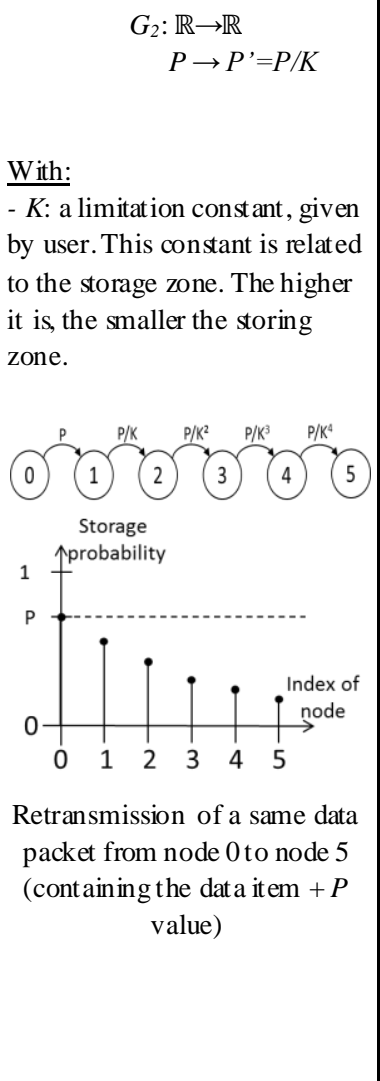 \\
\hline
\end{tabular}

In the case of a localized storage, the storage zone must be reduced to an area centered on the master node. As a result, for each approach, a limitation function $G$ is defined, which adapts the dissemination level (previously computed by the master node using $F$ ) to the storage zone. This function is executed on each node receiving a data item (see table 2).

\section{1) Hop-counter-based storage}

The master node starts broadcasting the packet with a counter value $H o p$ equal to $H_{0}$, computed with the conversion function $F_{1}$ from the importance level (see table 2). The packet is broadcasted from one node to all its neighbors, and at each hop the counter is decremented (Hop $\leftarrow H o p-1)$ until it reaches zero. In this case, the node must store the data, then resets $\mathrm{Hop}$ to its initial value $\left(H_{0}\right)$, and rebroadcasts the packet within its neighborhood. This process continues until the edges of material are reached. Note that $H_{0}$ is also included in the packet.

Our dissemination algorithm is broadcast based. With such communication model, the packet is further processed by nodes that have already received it. Furthermore, user can disseminate many segments or other data items. So, each packet is identified in the network layer (Packet $I D$ ) and then the nodes are limited to accept the same message only once. If a node receives again a message with the same $I D$, it will be dropped. Figure 5 shows the flowchart of this process executed in each node.

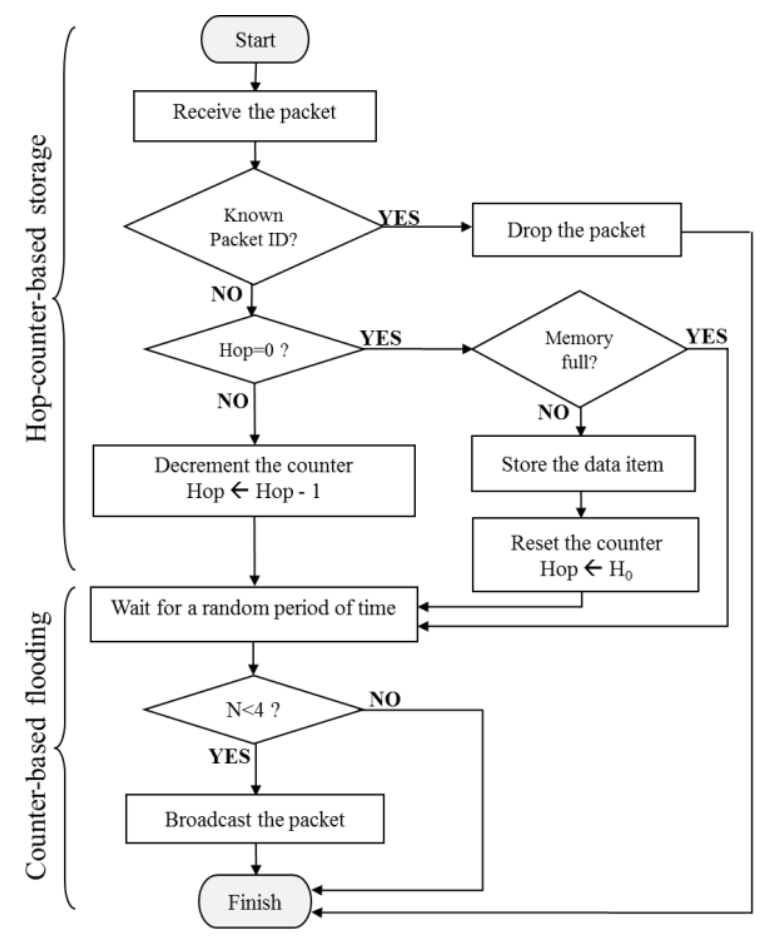

Fig. 5. Flowchart of hoop-counter dat a dissemination process.

\section{2) Probabilistic-based storage}

In this solution, data packets are flooded throughout the whole material and reach all sensor nodes using the counterbased flooding process. Each node decides to locally store data with the probability $P$ determined by the conversion function. As an example, let $P=0.5$. Upon receiving a data packet, a node selects a random value rand within the interval $[0,1]$. The node stores the data item only if $\operatorname{rand} \leq P(=0.5)$. Figure 6 shows the flowchart describing this process executed in each node. Only 
the storage part is illustrated, since the counter-based flooding is the same.

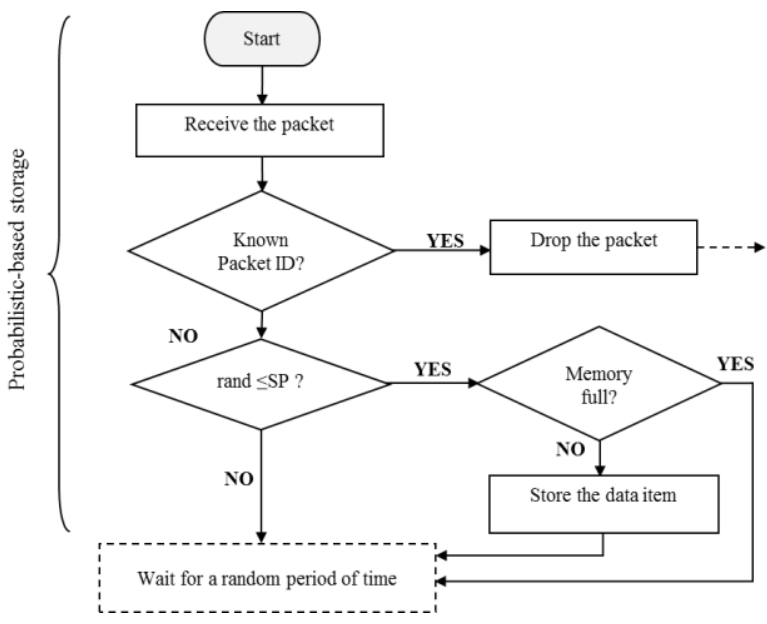

Fig. 6. Flowchart of probabilistic data dissemination process.

\section{Localized storage strategy}

Both strategies can be completed with limitation functions defined to restrain the storage zone around the master node. For the hop-counter-based approach, $G_{1}$ is defined as a piecewise linear function, that influences the value of $H_{0}$ (see table 2). Figure 7 describes the effect of the limitation function on $H_{0}$. Indeed, the data packet containing the data item is first sent from the master node $\left(n^{\circ} 0\right)$ to the network sensor. When the number of replications $N R$ (number of times the data item has been stored) becomes higher than $\operatorname{MaxNR}$, then the value of $H_{0}$ is changed to MaxHop. This new value thus prevents any other sensors to store the data item contained in this data packet.

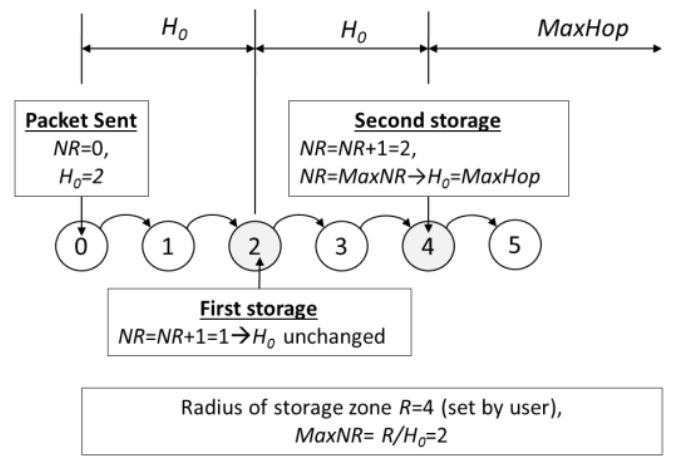

Fig. 7. Effect of the limitation function on the $H_{0}$ value

For the probability-based approach, the limitation function $G_{2}$ is a division of the initial probability by a constant $K$ (see table 2). Node after node, the probability decreases, moving closer to 0 , which prevents nodes to store the data item.

\section{SIMULATION ANDPERFORMANCE EVALUATION}

In this section, the simulation setup is described firstly. Then, the simulation results are presented and discussed.

\section{A. Simulation setup}

The performance of our solution is evaluated through simulation. Castalia/OMNET++ simulator is used to implement our dissemination algorithms. Currently, many wireless sens or network simulators are available as COOJA and TOSSIM but Castalia provides realistic wireless channel, radio models, and node behavior [26]. Nevertheless, Castalia is modified to support communicating material applications. A specific radio module is developed which is consistent with CC2420 components but allows more reduced transmission range. Thus, a thous and of scattered nodes in small material size could be simulated.

The simulated communicating material consists of 2500 nodes. The nodes positions are all uniformly distributed within a $5 \mathrm{~m} \times 5 \mathrm{~m}$ square $\left(100\right.$ nodes $\left./ \mathrm{m}^{2}\right)$ and the transmission power $-90 \mathrm{dbm}$ is used which ensures that a given node can only communicate with its direct neighbors. The chosen simulation parameters are summarized in table 3.

TABLE III. SIMULATION PARAMETERS

\begin{tabular}{|c|c|}
\hline Material size & $5 \mathrm{~m} \times 5 \mathrm{~m}$ \\
\hline Number of nodes & 2500 \\
\hline Nodes density & 100 nodes $/ \mathrm{m}^{2}$ \\
\hline Nodes distribution & Grid 50x50 \\
\hline Location of master node & Center of the material \\
\hline MAC layer & TMAC \\
\hline Transmission power & $-90 \mathrm{dbm}$ \\
\hline Radio & $\begin{array}{c}\text { CC2420 (Frequency }=20 \mathrm{MHz} \\
\text { Modulation=PSK, Throughput }=250 \\
\text { kbps) }\end{array}$ \\
\hline Radio Collision Model & Additive interference model \\
\hline
\end{tabular}

\section{B. Simulation results of non-localized dissemination}

\section{1) Non-segmented data item}

The impacts of the storage strategies on the uniformity performance for the proposed data dissemination algorithms have been investigated, through Castalia simulation. Figures 8 and 9 show the simulation results of the broadcast and storage mechanisms of hop-counter and probabilistic replication of a data item for different importance level (i.e. different hop and different probability, respectively).

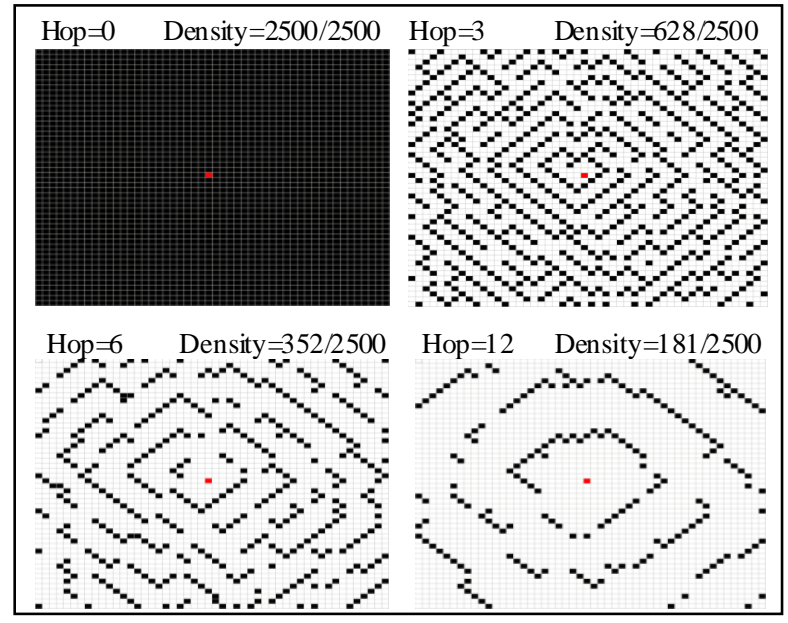

Fig. 8. Non-localized hop-count er storage of non-segmented data item.

On the basis of the obtained results, the following observation can be carried out. Both figures show the high reliability of the counter-based broadcasting scheme as all 
nodes store the information for $H o p=0$ and for $P=1$. Furthermore, the data is replicated in the communicating material according to the importance level (hop counter and probability of storage). When a low importance is used, for example $P=0.4$, data item is stored in 1000 nodes and less in the communicating material. The density of storage decreases with the importance level using the two algorithms.

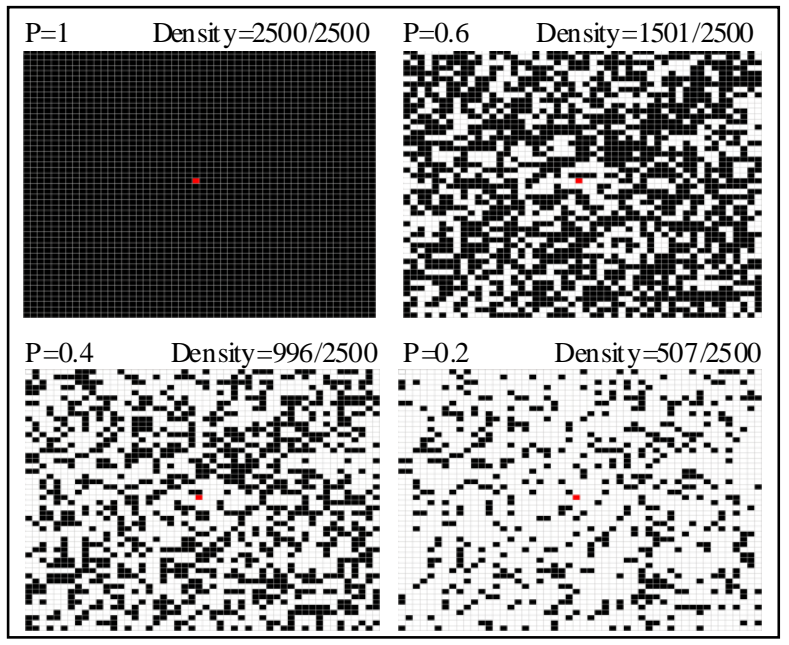

Fig. 9. Non-localized probabilistic storage of non-segmented data item.

To study the uniformity performance, the simulated material is divided into small cells of $50 \mathrm{~cm} \mathrm{x} 50 \mathrm{~cm}$ as shown in figure 10. Each piece has 25 sensor nodes as a uniform distribution (grid 50x50) is used. In each cell, the number of nodes that have stored the data item is counted.

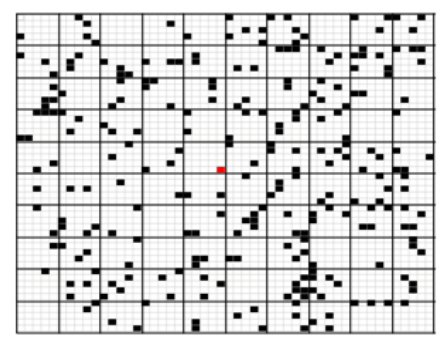

Fig. 10. Division of the material into pieces of $50 \mathrm{~cm} \times 50 \mathrm{~cm}$.

Figure 11 shows the storage density difference between cells, using the standard deviation tool. As the standard deviation presents the variable dispersing around its mean, the figure shows that the probabilistic algorithm has a resulting dissemination more uniform than the one obtained with the hop-counter approach.

When low importance is used (0.5 and less), the standard deviation decreases for the hop-counter algorithm (i.e. the uniformity is worst with large counter value). In that case, there are many pieces that are completely empty and others are poorly filled. However, there is no significant decrease of standard deviation for the probabilistic-based strategy because data item is uniformly replicated and many pieces are filled for all the importance level. Therefore, the hop-counter strategy produces more empty areas in the communicating material than the probabilistic-based in case of low importance level which could lead to data loss during material transformation.

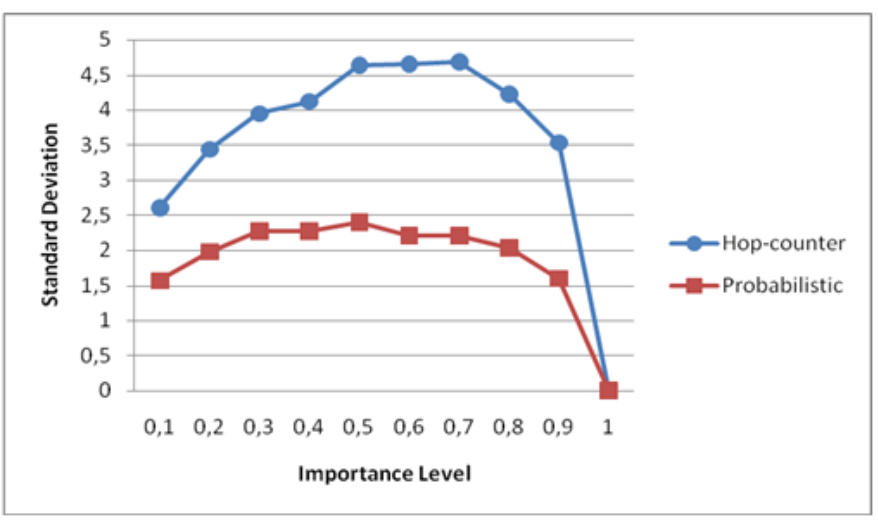

Fig. 11. Importance level versus standard deviation of storage strategies.

\section{2) Segmented data item}

Figures 12 and 13 show the simulation results of a segmented data item dissemination for the hop-counter and probabilistic strategies, respectively.

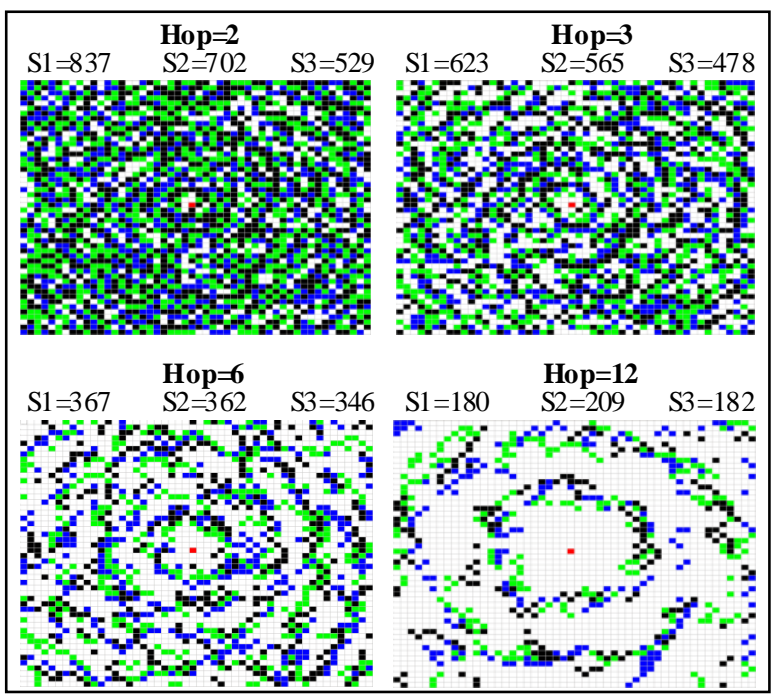

Fig. 12. Non-localized hop-counter storage of segmented data item.

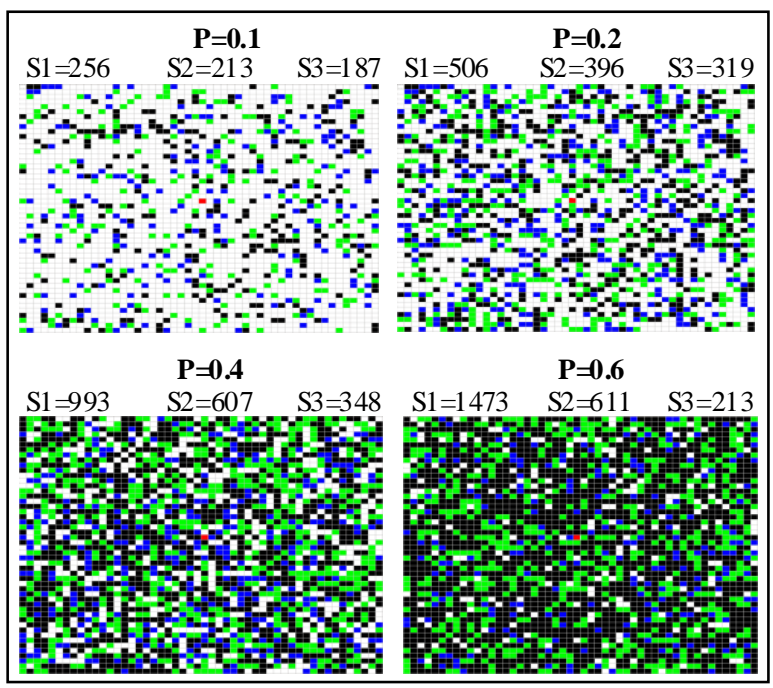

Fig. 13. Non-localized probabilist ic st orage of segmented data item. 
The three segments $(S 1, S 2$ and $S 3)$ are disseminated with the same hop-counter value (Hop) for the first algorithm and the same probability $P$ for the second one. Black, green, and blue colors present the nodes that have stored the first, second, and third segments, respectively.

Figure 12 shows that all the disseminated segments have the same density in the material for large hop-counter (Hop $=6$ and more) as the proposed algorithm tries to make exactly the same replicas of all broadcasted packets. However, like the non-segmented data items, there are more empty areas when the number of hops increases. Furthermore, if large size segments are disseminated with a low number of hops (e.g. $H o p=0$ ), the first broadcasted item is stored in all available memories of nodes and prevents the other segments to be replicated. As example, for Hop $=2$, the first segment is stored in more than 837 nodes, the second in 702 nodes and the last segment is replicated in 529 nodes.

This problem is more clear is figure 13 for probabilistic replication strategy. The first segment is replicated in a large number of nodes than the others for high importance level which present a deficiency problem of memory space. As example for storage probability $P=0.6$, the first segment is replicated in 1473 nodes and the last one is stored in 213 nodes.

A solution to these problems could rely on a dynamic selforganization of the data item storage, in which node autonomously decides to replicate or not. The replication process may uses a storage control approach similar to neighbomood negotiation to know if the data has already been stored by other nodes [19], or applying data compression techniques such as those described in [27] [28]. Compression algorithms are suited for reduced storage and limited resources of ultra-s mall nodes (e.g. in [27] compression ratios up to $70 \%$ on environmental datasets).

\section{Simulation results oflocalized dissemination}

\section{1) Non-segmented data item}

Simulation results of localized data item dissemination are shown in figures 14 and 15 for hop-counter $(R=6)$ and probabilistic approaches $(K=2.5)$, respectively.

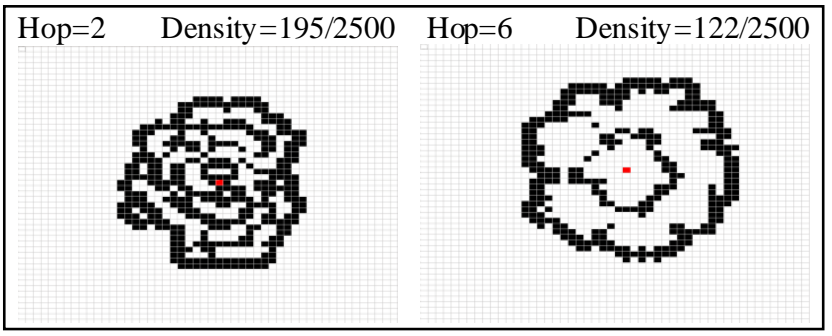

Fig. 14. Localized hop-counter storage of non-segmented data item.

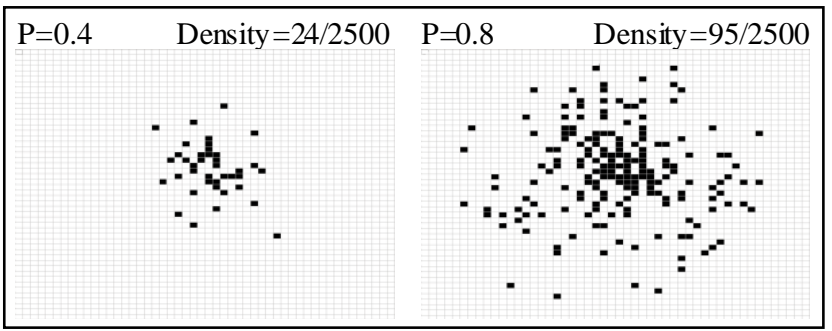

Fig. 15. Localized probabilistic storage of non-segmented data item.
As shown in figure 14, it can be concluded that the hop-byhop replication mechanism guarantees efficient data item storage in the target area of the material. The storage density is increased according to the hop-counter, as example, for Hop=2 data item is replicated in 195 nodes. However, for Hop $=6$, it is stored in 122.

As for probabilistic approach, the data is also efficiently replicated in the desired area as shown in figure 15 . The density of storage is high in nodes around the master (i.e. the probability $P$ is high in that nodes and it decreases away from the master).

\section{1) Segmented data item}

Simulation results of localized segmented data item dissemination are shown in figures 16 and 17 for hop-counter and probabilistic approaches, respectively.

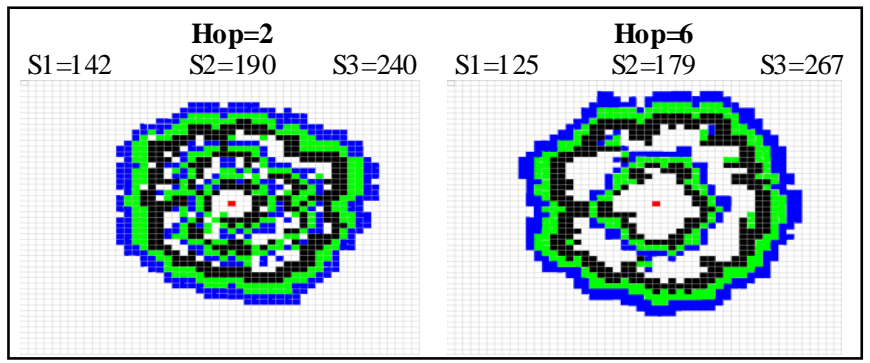

Fig. 16. Localized hop-counter storage of segmented data item.

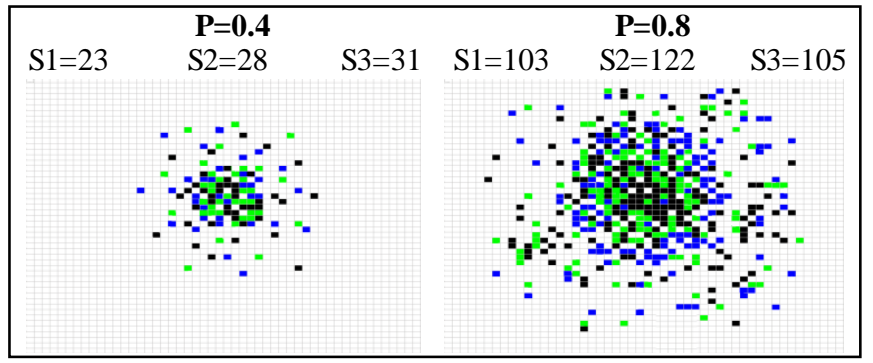

Fig. 17. Localized probabilistic storage of segmented data item.

The master node starts by disseminating the first segment. If the node memory is full, the node retransmits the packet without decrementing the counter. That is why the second segment is replicated outside the first one and the same for third segment as shown in figure 16. The last broadcasted item is replicated in large number of node than others. As example for $H o p=6$, the first segment is stored in 125 nodes, the second in 179 and the last segment is replicated in 267 nodes.

However, the segments are stored in the target area with the same replication rate using the probabilistic algorithm as shown in figure 17. All the segments have the same density. For this reason, probabilistic approach is more efficient than hop-counter for localized dis semination.

\section{CONCLUSION}

In this paper, data dissemination algorithms are proposed to store information in communicating materials through embedded ultra-small wireless sensor nodes. A counter-based flooding approach coupled with storage strategies is used to disseminate the packets and to control the replication rate according to the importance level of the user information. Nonlocalized and then localized storage algorithms are studied. For 
each storage mode, hop-counter and probabilistic replication strategies are proposed. The performances of these solutions are evaluated through computer simulation for non-segmented and segmented data. For non-localized dissemination, simulation results show that our probabilistic algorithm provides a more uniform replication than hop-counter-based which can more avoid data loss during physical material transformation. For localized dis semination, simu lation results show an efficient localized storage for non-segmented data using both the hop counter and the probabilistic approaches . However, probabilistic algorithm outperforms the other for segmented item as all the segments are replicated in the target area with the same density.

\section{REFERENCES}

[1] S. Kubler, W. Derigent, A. Thomas, E. Rondeau,"Problem definition methodology for the Communicating Material paradigm", 10th IFAC Workshop on Intelligent Manufacturing Systems, 1-2 July 2010, Lisbonne, Portugal.

[2] S. Kubler, W. Derigent, A. Voisin, A. Thomas, E. Rondeau, "Method for embedding context-sensitive information on "communicating textiles" via fuzzy AHP", Journal of Intelligent and Fuzzy Systems, February 2013.

[3] S. Kubler, W. Derigent, A. Thomas, E. Rondeau, "Embedding data on "communicating materials" from context-sensitive information analysis", Journal of Intelligent Manufacturing, March 2013.

[4] M.U. Mahfuz, K.M. Ahmed, "A review of micro-nano-scale wireless sensor networks for environmental protection: Prospects and challenges", Science and Technology of Advanced Materials, Vol. 6, No. 4, May 2005, pp. 302-306.

[5] M. Akdere, C.C. Bilgin, O. Gerdaneri, I. Korpeoglu, O. Ulusoy, U. Cetintemel, "A comparison of epidemic algorithms in wireless sensor networks", Journal of Computer Communications, Vol. 29, August 2006, pp. 2450-2457.

[6] F. Hongping, F. Kangling, "Overview of data dissemination strategy in wireless sensor networks", International Conference on E-Health Networking, Digital Ecosystems and Technologies, 17-18 April 2010, Shenzhen, China, pp. 260-263.

[7] Y. Busnel, A. Ghodsi, K. Iwanicki, H. Miranda, H. Weatherspoon, "Gossiping over storage systems is practical", ACM SIGOPS Operating Systems Review, Vol. 41, No. 5, 2007, pp. 75-81.

[8] O. Sekkas, D. Piguet, C. Anagnostopoulos, D. Kotsakos, G. Alyfantis, C. Kassapoglou-Faist, S. Hadjiethymiades, "Probabilist ic information dissemination for MANETs: the IPAC approach", 20th Tyrrhenian Workshop on Digital Communication, 2010, Pula, It aly,pp. 375-385.

[9] P. Kyasanur, R. Choudhury, I. Gupta, "Smart gossip: an adaptive gossipbased broadcasting service for sensor networks", IEEE International Conference on Mobile Ad Hoc and Sensor Systems, Octobre 2006, Vancouver, British Columbia, Canada, pp. 91-100.

[10] H. Miranda, S. Leggio, L. Rodrigues, K. Raatikainen, “A power-aware broadcasting algorithm", 17th IEEE International Symposium on Personal, Indoor and Mobile Radio Communications, 11-14 September 2006, Helsinki, Finland, pp. 1-5.

[11] C. Zhi-yan, J. Zhen-zhou, H. Ming-zeng, "An energy-aware broadcast scheme for directed diffusion in wireless sensor network", Journal of Communication and Computer, Vol. 4, No. 5, May 2007, pp. 28-35.

[12] E.R. Sanchez, M. Rebaudengo, L. Zhang, "Performance evaluation of reliable and unreliable opportunistic flooding in wireless sensor network", 17th IEEE International Conference on Networks, 14-16 December 2011, Singapore, pp. 7-12.
[13] B. Garbinato, D. Rochat, M. Tomassini, F. Vessaz, "Injecting powerawareness into epidemic information dissemination in sensor networks", Journal of Future Generation Computer Systems, Vol. 26, 2010, pp. 868-876.

[14] S. Izumi, T. Matsuda, H. Kawaguchi, C. Ohta, M. Yoshimoto, "Improvement of Counter-based Broadcasting by Random Assessment Delay Extension for Wireless Sensor Networks", IEEE International Conference on Sensor Technologies and Applications, 14-20 October 2007, Valencia, Spain, pp. 76-81.

[15] S. S. Kulkarni, L. Wang, "MNP: Multihop Network Reprogramming Service for Sensor Networks", 25th IEEE International Conference on Distributed Computing Systems, June 2005, Columbus, Ohio, USA, pp. 7-16.

[16] W. Dong, C. Chen, X. Liu, G. Teng, J. Bu, Y. Liu, "Bulk data dissemination in wireless sensor networks: Modeling and analysis", Computer Networks, Vol. 56, 2012,pp. 2664-2676.

[17] G. Maia, D.L. Guidoni, A.C. Viana, A.L. Aquino, R.A. Mini, A.A. Loureiro, "A distributed data storage protocol for heterogeneous wireless sensor networks with mobile sinks", Ad Hoc Networks, Vol. 11, No. 5, July 2013, pp. 1588-1602.

[18] J. Neumann, N. Hoeller, C. Reinke, V. Linnemann, "Redundancy infrastructure for service-oriented wireless sensor networks", 9th IEEE International Symposium on Network Computing and Applications,1517 July 2010, Cambridge, Massachusetts,USA, pp. 269-274.

[19] P. Gonizzi, G. Ferrari, V. Gay, J. Leguay, "Data dissemination scheme for distributed storage for IoT observation systems at large scale", Journal of Information Fusion, April 2013.

[20] M. Vecchio, A. C. Viana, A. Ziviani, R. Friedman, "DEEP: Densitybased proactive dat a dissemination protocol for wireless sensor net works with uncontrolled sink mobility", Journal of Computer Communicat ions, Vol. 33, No. 8, 2010, pp. 929-939.

[21] Z. Bar-Yossef, R. Friedman, G. Kliot, "RaWMS - RandomWalk based Light weight Membership Service forWireless Ad Hoc Networks", ACM Transactions on Computer Systems, Vol. 26, No. 2, 2008, pp. 1-66.

[22] Y. Tseng, S. Ni, E. Shih, "Adaptive Approaches to Relieving Broadcast Storms in a Wireless Multihop Mobile Ad Hoc Network", IEEE Transactions on Computers, Vol. 52, No. 5, May 2003, pp. 545-557.

[23] C. Chen, C. Hsu, H. Wang, "DISCOUNT: A Hybrid Probability-Based Broadcast Scheme for Wireless Ad Hoc Networks", 62nd IEEE Conference on Vehicular Technology, Vol. 4, 25-28 September 2005, pp. 2706-2710.

[24] J. Arango, A. Efrat, S. Ramasubramanian, M. Krunz, S. Pink, "Retransmission and Back-off Strategies for Broadcasting in Multi-hop Wireless Networks", 3rd IEEE International Conference on Broadband Communication, Networks and Systems, 1-5 October 2006, San José, California, USA.

[25] S. Izumi, T. Takeuchi, T. Matsuda, H. Kawaguchi, C. Ohta, M. Yoshimoto, "Counter-Based Broadcasting with Hop Count Aware Random Assessment Delay Extension for Wireless Sensor Networks", IEICE Transactions on Communications, Vol.E91-B, No.11, November 2008, pp.3489-3498.

[26] H. Sundani, H. Li, V. Devabhaktuni, M. Alam, P. Bhattacharya, "Wireless Sensor Network Simulators, A Survey and Comparisons", International Journal of Computer Networks, Vol. 2, No. 5, 2011, pp. 249-265.

[27] F. Marcelloni, M. Vecchio, "A simple algorithm for data compression in wireless sensor net works", IEEE Communications Letters, Vol. 12, No. 6, June 2008, pp. 411- 413.

[28] C.M. Sadler, M. Martonosi, "Data compression algorithms for energyconstrained devices in delay tolerant networks", Fourth International Conference on Embedded Networked Sensor Systems, 1-3 November 2006, Boulder, Colorado, USA, pp. 265-278. 